\title{
Thermal quenching properties of ultraviolet emitting centers in mesoporous silica
}

\author{
C. M. Carbonaro, ${ }^{1, *}$ P. C. Ricci, ${ }^{1}$ and A. Anedda ${ }^{1,2}$ \\ ${ }^{1}$ Department of Physics, University of Cagliari, s.p. 8, km 0.700, 09042 Monserrato, Italy \\ ${ }^{2}$ LIMINA Laboratory, University of Cagliari, s.p. 8, km 0.700, 09042 Monserrato, Italy
}

(Received 15 January 2007; revised manuscript received 18 June 2007; published 27 September 2007)

\begin{abstract}
We report an investigation of the thermal quenching properties of the ultraviolet emission observed in mesoporous silica. The optical emission is due to the contribution of two emitting centers at 3.7 and $4.0 \mathrm{eV}$ and previously assigned to strong- and weak-interacting silanols $(\mathrm{Si}-\mathrm{OH})$ located at the surface of the material. We investigate the temperature dependence of the lifetimes and the photoluminescence amplitudes of the two centers, in the 10-300 K range, by exciting in the ultraviolet and vacuum ultraviolet region with synchrotron radiation. The analysis of the lifetimes, carried out in the framework of a biexponential model at all the temperatures and excitations, and of the temperature dependence of the photoluminescence amplitude displayed different quenching properties in samples with different pore diameters. In addition, the thermal dependence of the photoluminescence amplitudes shows a distribution of the activation energy as a function of the emission energy in samples with smaller pore diameter.
\end{abstract}

DOI: 10.1103/PhysRevB.76.125431 PACS number(s): 78.55.Mb, 61.46. $-\mathrm{w}$, 78.47.+ p, 81.07.-b

\section{INTRODUCTION}

The visible and ultraviolet photoluminescence (PL) properties observed in silicon and silica-based nanostructured materials have gathered a large and increasing interest in the last decade because of their possible applications in optoelectronics. ${ }^{1}$ Beside the technological interest, the topic is also relevant from a basic research point of view, since the attribution of these optical features to quantum confinement effects or to defect-related mechanisms is still debated. ${ }^{1-6}$ Concerning porous and nanocrystalline silicon, the emissions in the red to near-infrared region $(2.1-1.3 \mathrm{eV})$ depend on the dimensions of the $\mathrm{Si}$ nanocrystals, providing experimental evidence of the role of the quantum confinement effects. ${ }^{1,2}$ On the other hand, blue-green emission is ascribed to surface-related defects, including both $\mathrm{C}$ or $\mathrm{OH}$ groups adsorbed at the surface of the porous material. ${ }^{1-6}$ As regards silica-based nanostructered materials, red, green, and blue emissions from silica nanoparticles, mesoporous silica, and silica nanotubes were reported with strong similarities with the spectroscopic features observed in nanoscaled silicon. ${ }^{7-10}$ Since the thickness of the bulk silica walls in nanostructured silica materials $[\sim 1 \mathrm{~nm}$ (Ref. 11)] is comparable to the thickness of oxide layers which cover silicon in nanoscaled silicon materials, these similarities can help to clarify the debated origin of the observed features. The blue emission reported in silica nanoparticles at about $2.7 \mathrm{eV}$ is attributed to self-trapped excitons whose spectral features are affected by the nanoscale of the silica particles studied. ${ }^{12-14}$ Experimental evidence was reported to claim the attribution of the green luminescence to $\mathrm{H}$ - or $\mathrm{OH}$-related surface defects and the attribution of the red emission to surface nonbridging oxygen. ${ }^{15-17}$ Beside the PL bands in the visible range, few articles have dealt with ultraviolet (UV) emissions observed in silicon oxide films, oxidized porous silicon, and mesoporous silica. ${ }^{18-23}$ In a recent study focused on the emission peaked at about $3.7 \mathrm{eV},{ }^{22-24}$ we reported experimental evidence for the presence of two distinct surface centers emitting in the UV range whose spectral features are summarized by the schematic picture shown in Fig. 1. The two PL bands have emission peaks at 3.7 and $4.0 \mathrm{eV}$, two main excitations at 5.4-6.2 eV and 5.7-6.5 eV, respectively, and estimated time decays of about 20 and 2 ns.

The observation of different silanol groups $(\mathrm{Si}-\mathrm{OH})$ at the surface of the examined porous silicas ${ }^{25}$ and the dependence of the UV bands on the content of $\mathrm{OH}$ groups ${ }^{18,24}$ led to the attribution of the reported spectroscopic features to two species of interacting silanols, strong-interacting silanols (IS-1, $\mathrm{PL}$ at $3.7 \mathrm{eV}$ ) and weak-interacting silanols (IS-2, PL at $4.0 \mathrm{eV}) .{ }^{23}$ However, other models are still possible where the sylanol groups are responsible for the UV emission in an indirect way. It was recently reported that the blue emission in silicon nanostructure is due to a defect pair consisting of a dioxasilirane center and a silylene center formed by the de-

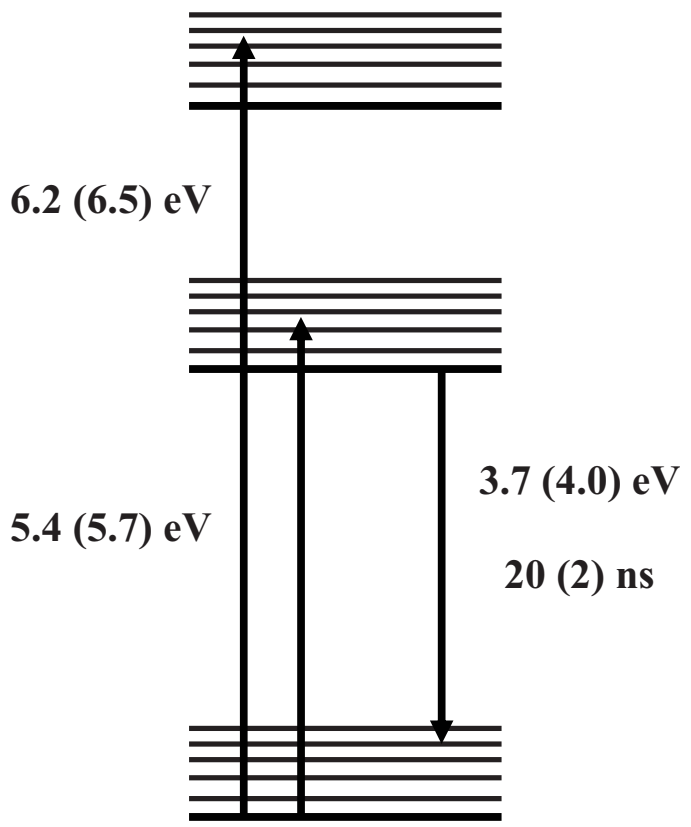

FIG. 1. Electronic energy level scheme for the IS-1 (IS-2) centers. 
hydroxylation of surface silanol groups. ${ }^{6}$ The dependence of water content or aging condition of the blue band in silicon nanostructure strongly recalls that of the UV bands in porous silica, suggesting a dehydroxylationlike model also for these centers, where the interacting silanol groups are regarded as indirectly responsible for the UV emissions. Since the contribution of different types of interacting silanols depends on the porosity of the samples and the two centers showed very similar spectroscopic properties, it is interesting to investigate possible energy transfer or conversion mechanisms between the two species. Indeed, the contribution of the two PL bands to the steady-state emission at room temperature and $10 \mathrm{~K}$ is different in samples with small $(3.2 \mathrm{~nm})$ and large $(18.2 \mathrm{~nm})$ pore diameters. ${ }^{23}$ In the first case the contribution of IS-1 with respect to that of IS-2 increases (about four times) by decreasing the temperature; in the second case a slight decrease is reported. The increase of IS- 1 centers with respect to IS-2 centers is expected because of the shrinking of the glassy matrix at low temperature; on the contrary, the increase of the relative contribution of IS-2 in samples with larger pores should be related to a different mechanism. This aspect is investigated in the present work by studying the amplitude and the time decay of the PL at 3.7 and $4.0 \mathrm{eV}$ as a function of the excitation energy and the temperature in the range $10-300 \mathrm{~K}$ in mesoporous silica with mean pore diameter of 3.2 and $18.2 \mathrm{~nm}$.

\section{EXPERIMENTAL SECTION}

Measurements were performed on sol-gel synthesized commercial porous silica monoliths (diameter $5 \mathrm{~mm}$, thickness $2 \mathrm{~mm}$ ) produced by Geltech Inc. ${ }^{26}$ Investigated samples have pore diameter of 18.2, samples A, and $3.2 \mathrm{~nm}$, samples B [Brunauer-Emmett-Teller (B.E.T.) analysis, 5\% of standard deviation], specific surface area of 264 and $594 \mathrm{~m}^{2} / \mathrm{g}$, and density of 0.6 and $1.2 \mathrm{~g} / \mathrm{cm}^{3}$. Samples were analyzed as prepared; no chemical and/or physical treatment was carried out to remove the water contained within the porous structure.

PL measurements were carried out at the SUPERLUMI experimental station on the I beamline of the HASYLAB synchrotron laboratories at Desy (Hamburg) by using the pulsed excitation light of the synchrotron radiation (SR). The PL signal was dispersed by a Spectra-Pro 300i monochromator and detected in the $1.5-5.0 \mathrm{eV}$ energy range with a photomultiplier (Hamamatsu 206358P). The spectral emission bandwidth was $11 \mathrm{~nm}$. PL spectra were recorded under multibunch operation and detected with an integral time window of 192 ns correlated to the SR pulses. Time decays in the nanosecond domain were gathered under single-bunch operation, using 1024 channels to scan the 192-ns interval time between adjacent pulses [pulse width of $0.2 \mathrm{~ns}$ (Ref. 27)]. A continuous-flow liquid helium cryostat was used to set the temperature of the sample chamber in the $10-300 \mathrm{~K}$ range, with an accuracy of $\pm 1 \mathrm{~K}$.

\section{RESULTS}

Figure 2 reports the time decay of the PL at $4.0 \mathrm{eV}$ excited at $6.53 \mathrm{eV}$ in samples $\mathrm{A}$ at three different temperatures

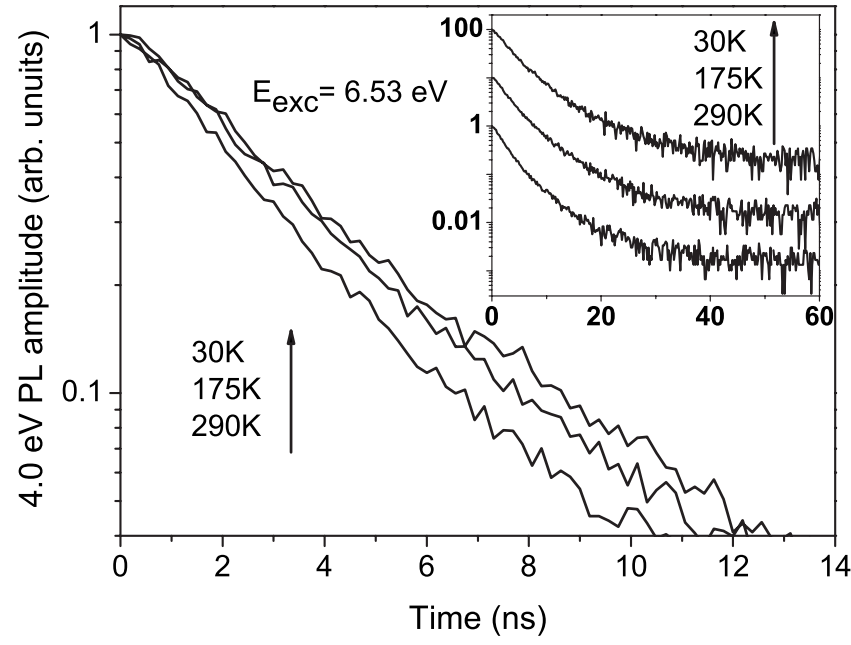

FIG. 2. Time decay of the PL at $4.0 \mathrm{eV}$ excited at $6.53 \mathrm{eV}$ at three different temperatures $(30,175$, and $290 \mathrm{~K})$ in samples A. The inset shows the time decays over a larger time scale.

(30, 175, and $290 \mathrm{~K})$. The dynamic range of the PL amplitude at each temperature in the whole explored range is of about three orders of magnitude, allowing a fine characterization of the decay profile. As expected, the time decay increases as the temperature decreases because of the smaller contribution to the time decay of nonradiative recombinations. The semilogarithmic scale of the inset displays that the time decay cannot be expressed by a single-exponential law at every investigated temperature. Analogous results are observed by monitoring the time decay of the PL band at $3.7 \mathrm{eV}$ and for both emissions under excitation at $5.64 \mathrm{eV}$ also in samples B. The nonsingle-exponential character of the time decays is also evidenced by comparing the time decay of the emissions of samples A under the same excitation energy [Fig. 3(a)] and the time decay of a selected emission under excitation at 5.64 and $6.53 \mathrm{eV}$ [Fig. 3(b)]. The nonsingle-exponential character is more pronounced by monitoring the $3.7-\mathrm{eV}$ band than the $4.0-\mathrm{eV}$ band under excitation at $6.53 \mathrm{eV}$ while no differences between the time decays collected under the $5.64 \mathrm{eV}$ excitation are observed (not shown in the figure). In a similar way, the nonsingle exponential character is more pronounced gathering the emission at $3.7 \mathrm{eV}$ excited at $6.53 \mathrm{eV}$ instead of $5.64 \mathrm{eV}$ and no differences are reported for the emission at $4.0 \mathrm{eV}$ (not shown in the figure). Data reported in Fig. 3 refer to $10 \mathrm{~K}$, but the results hold in the whole range of investigated temperatures. Figure 4 reports analogous results for samples B: the nonsingle exponential character increases as the excitation energy [Fig. 4(a)] or the temperature [Fig. 4(b)] increase.

In Figs. 5 and 6 the temperature dependence of the PL amplitude in samples A and B is reported. The PL amplitude at 3.7 and $4.0 \mathrm{eV}$, excited at 6.53 and $5.39 \mathrm{eV}$, respectively, in samples $\mathrm{A}$ is quenched as the temperature increases. Analogous results are recorded for samples B by collecting the PL amplitude at $3.7 \mathrm{eV}$ excited at $5.64 \mathrm{eV}$. Data are fitted according to an one-process quenching model (see Discussion), and no distribution of the activation energy is observed but in samples B (inset of Fig. 6; solid line is a guide for the eyes). 

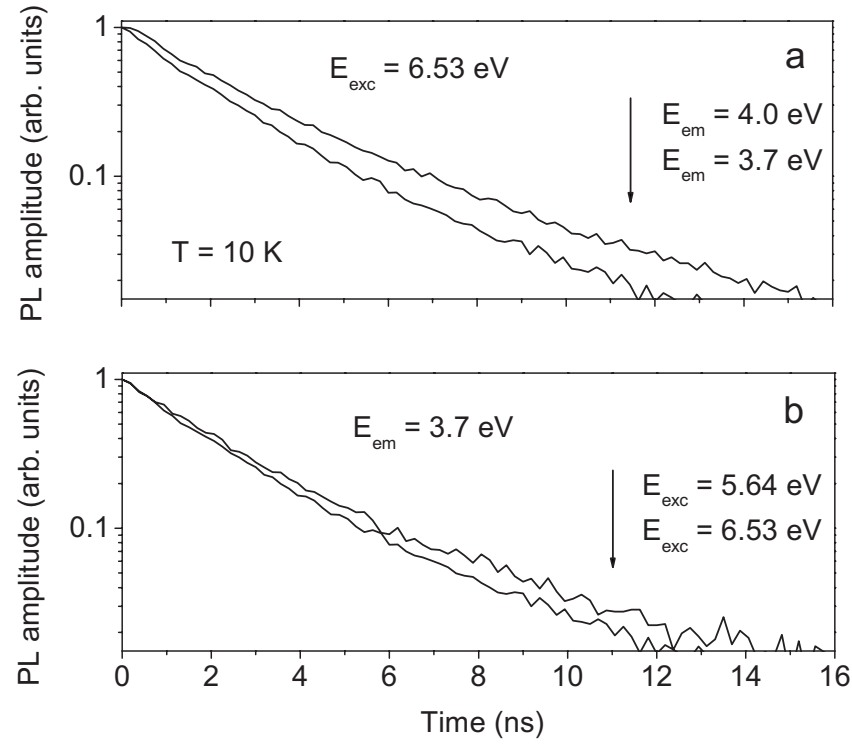

FIG. 3. Time decay of the 3.7 and $4.0 \mathrm{eV}$ emissions under $6.53 \mathrm{eV}$ excitation (a) and time decay of $3.7 \mathrm{eV}$ emission under excitation at 5.64 and $6.53 \mathrm{eV}$ (b) in samples $\mathrm{A}$ at $\mathrm{T}=10 \mathrm{~K}$.

\section{DISCUSSION}

The task of the present work is the investigation of the thermal quenching properties of UV-emitting centers in porous silica by comparing the temperature dependence of the time decay and the PL amplitude. The reported results confirm that the nonsingle-exponential character of the UV emission is observed in the whole temperature range from room temperature down to $10 \mathrm{~K}$ and is enhanced by exciting at about $6.5 \mathrm{eV}$.

Following the hypothesis of two emitting centers previously reported, ${ }^{22,23}$ the time decay is fitted with a biexponential law 28

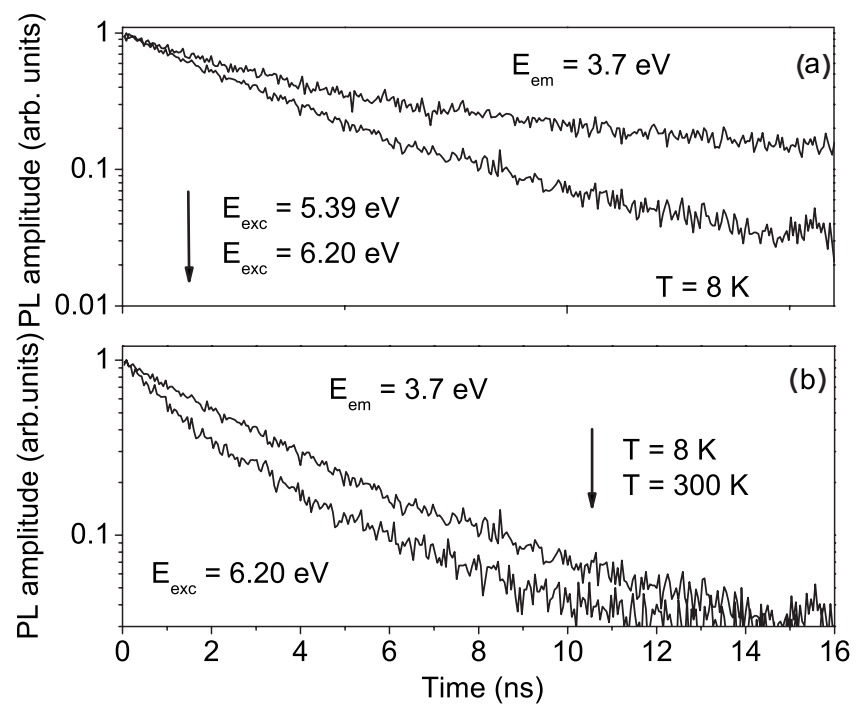

FIG. 4. Time decay of the $3.7-\mathrm{eV}$ band excited at 5.39 and $6.20 \mathrm{eV}$ at $8 \mathrm{~K}$ (a) and excited at $6.20 \mathrm{eV}$ at 8 and $300 \mathrm{~K}$ (b) in samples B.

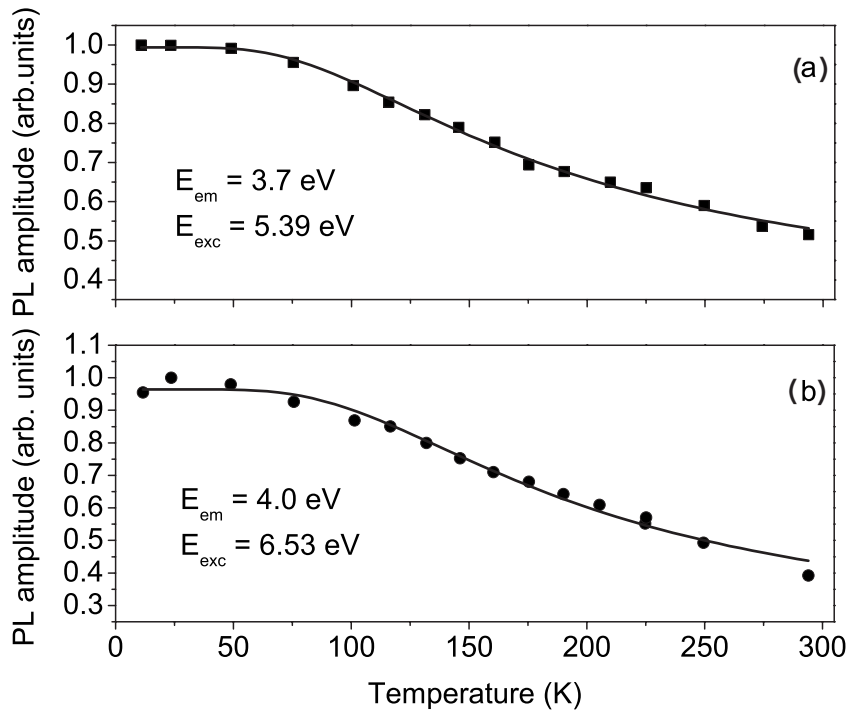

FIG. 5. Temperature dependence of the PL amplitude at 3.7 and $4.0 \mathrm{eV}$ excited at $5.39 \mathrm{eV}$ (a) and $6.53 \mathrm{eV}$ (b), respectively, in samples A.

$$
I=a_{0}+a_{1} \exp \left(-t / \tau_{1}\right)+a_{2} \exp \left(-t / \tau_{2}\right),
$$

where $I$ is the PL amplitude, $\tau_{1}$ and $\tau_{2}$ are the characteristic lifetimes of the two emitting centers, $a_{1}$ and $a_{2}$ the preexponential factors, and $a_{0}$ is a constant base line. The fit procedure was successfully applied to all the data (square correlation factor $R^{2} \geqslant 0.99$ ) for both the two emissions and excitations at all temperatures. In the whole cases the fit indicated a fast time decay of about $2-3 \mathrm{~ns}$ and a slow time decay of about 15-20 ns. In Fig. 7(a) is reported, as an example, the time decay of the $4.0-\mathrm{eV}$ PL band excited at $6.53 \mathrm{eV}$ in samples A at $215 \mathrm{~K}$; the solid line is the result of the fitting procedure. At all temperatures, the larger contribution to the time decay is due to the fast component as indi-

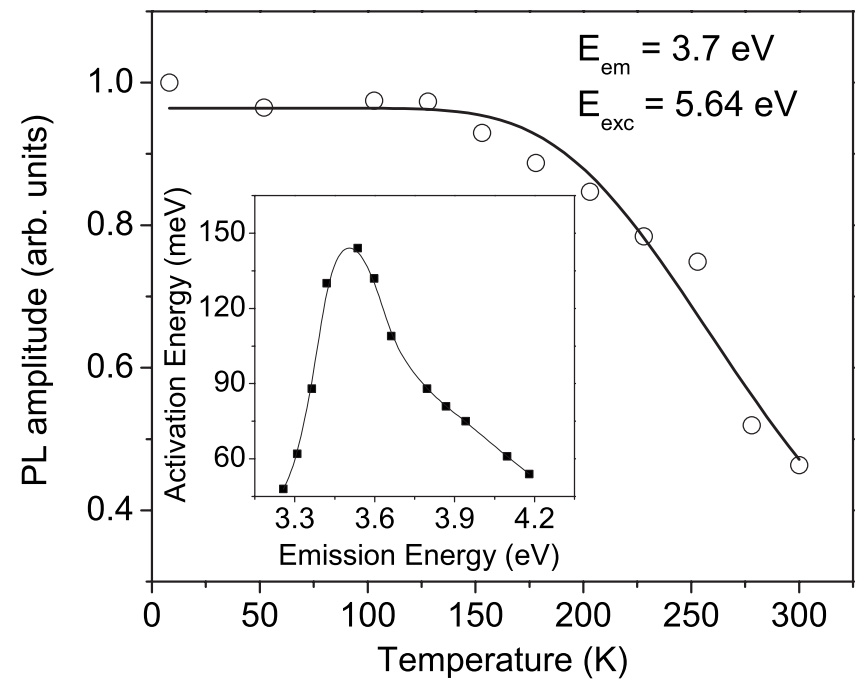

FIG. 6. Temperature dependence of the PL amplitude at $3.7 \mathrm{eV}$ excited at $5.64 \mathrm{eV}$ in samples $\mathrm{B}$. The inset shows the distribution of the calculated activation energy as a function of the emission energy (solid line is a guide for the eyes). 


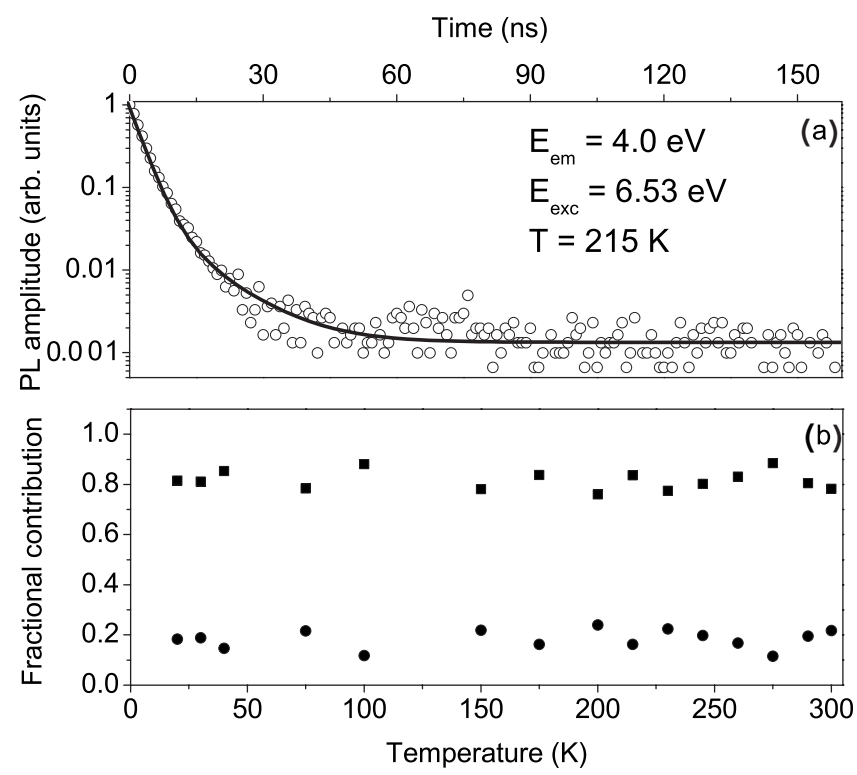

FIG. 7. (a) Time decay of the $4.0-\mathrm{eV}$ band under $6.53 \mathrm{eV}$ excitation at $215 \mathrm{~K}$ in samples $\mathrm{A}$. The solid line is the fitting result according to the biexponential model. (b) Fractional contributions of the two time decays as a function of the temperature (squares $f_{1}$, circles $f_{2}$ ).

cated by the fractional contributions of the two time decays as a function of the temperature reported in Fig. 7(b). In the framework of a biexponential model one can define an average lifetime as follows: ${ }^{28}$

$$
\bar{\tau}=\frac{a_{1} \tau_{1}^{2}+a_{2} \tau_{2}^{2}}{a_{1} \tau_{1}+a_{2} \tau_{2}}=f_{1} \tau_{1}+f_{2} \tau_{2},
$$

where

$$
f_{i}=\frac{a_{i} \tau_{i}}{\sum_{i} a_{i} \tau_{i}}
$$

The fractional contributions $f_{1}$ and $f_{2}$ indicate the relative weights of the two time decays to the lifetime. One should note, however, that the two fractional contributions are largely correlated, as indicated in Fig. 7(b). The mean contribution of the slow time decay $\left(f_{2}\right)$ is about $20 \%$ while the mean contribution of the fast time decay $\left(f_{1}\right)$ is about $80 \%$. Analogous results also hold for samples B and by changing the recorded emission and the excitation energy, but for the 3.7-eV PL excited at $5.64 \mathrm{eV}, f_{2}$ is about $30 \%$. Since the fractional contribution of the slow component to the time decay is small, its estimated lifetime is affected by a large variability (the estimated error is about $30 \%$ ). On the contrary the contribution of the fast component to the time decay is large and its estimated lifetime is affected by a smaller variability (the estimated error is about 10\%). For this reason the analysis of the temperature dependence of the time decay is carried out on the lifetime of the fast emission.

The lifetime is related to the radiative $\left(k_{r}\right)$ and nonradiative $\left(k_{n r}\right)$ decay rate, according to the expression

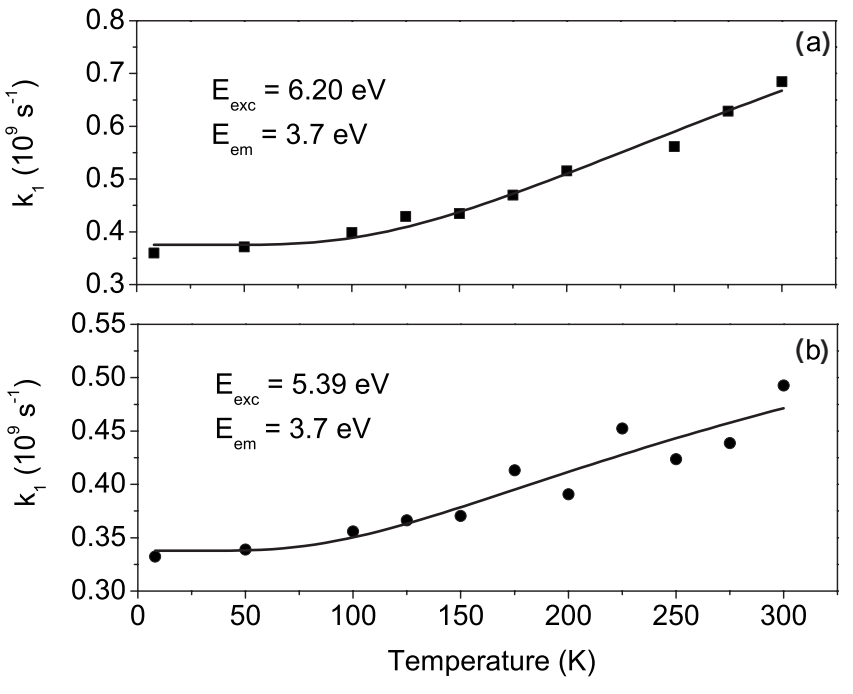

FIG. 8. Temperature dependence of the fast component decay rate as deduced by the biexponential model monitoring the emission at $3.7 \mathrm{eV}$ excited at $6.20 \mathrm{eV}$ (a) and $5.39 \mathrm{eV}$ (b) in samples B. Solid lines are fitting results according to Eqs. (4) and (5) (see text for details).

$$
\tau=\frac{1}{k}=\frac{1}{k_{r}+k_{n r}(T)} .
$$

The temperature dependence of the lifetime is due to the nonradiative decay rate and can be expressed as follows:

$$
k_{n r}(T)=A \exp \left(-E_{a} / k_{B} T\right),
$$

where $A$ is a preexponential factor, $E_{a}$ is the activation energy, and $k_{B}$ is the Boltzmann constant. The temperature dependence of the decay rate of the fast emission $\left(k_{1}\right)$ as deduced by the biexponetial fitting procedure is reported in Figs. 8 and 9 for samples B and A, respectively. The solid line is the result of the best-fitting procedure according to Eqs. (4) and (5). The temperature dependence of the decay rate of the fast emission deduced with the biexponential model by monitoring the emission at $3.7 \mathrm{eV}$ in samples $\mathrm{B}$ excited at $6.20 \mathrm{eV}$ [Fig. 8(a)] and $5.39 \mathrm{eV}$ [Fig. 8(b)] indicates an activation energy of $(40 \pm 5) \mathrm{meV}$ and $(31 \pm 9) \mathrm{meV}$ (square correlation factor $R^{2} \geqslant 0.98$ and 0.89 , respectively). Analogous result is obtained with samples A by monitoring the emission at $4.0 \mathrm{eV}$ excited at 6.53 and $5.63 \mathrm{eV}$, as reported in Fig. 9(a) for the first case $\left(E_{a}=40 \pm 20 \mathrm{meV}, R^{2}\right.$ $\geqslant 0.98$ ). On the contrary, by gathering the PL at $3.7 \mathrm{eV}$ in samples A a larger activation energy is obtained, both under excitation at $6.53 \mathrm{eV}$ [Fig. 9(b), $\left.E_{a}=90 \pm 50 \mathrm{meV}, R^{2} \geqslant 0.60\right]$ and $5.39 \mathrm{eV}$ (not shown for the sake of brevity, $E_{a}$ $\left.=170 \pm 90 \mathrm{meV}, R^{2} \geqslant 0.62\right)$, but with a smaller correlation factor. We conclude that the activation energy of the $4.0-\mathrm{eV}$ band is $30-40 \mathrm{meV}$, but we need to explain the results gathered by monitoring the $3.7-\mathrm{eV}$ emission.

The activation energy can also be calculated by analyzing the temperature dependence of the PL amplitude. As reported in Figs. 5 and 6 the UV emission decreases as the temperature increases. In order to distinguish between the contributions of the two emitting centers, the thermal quenching 

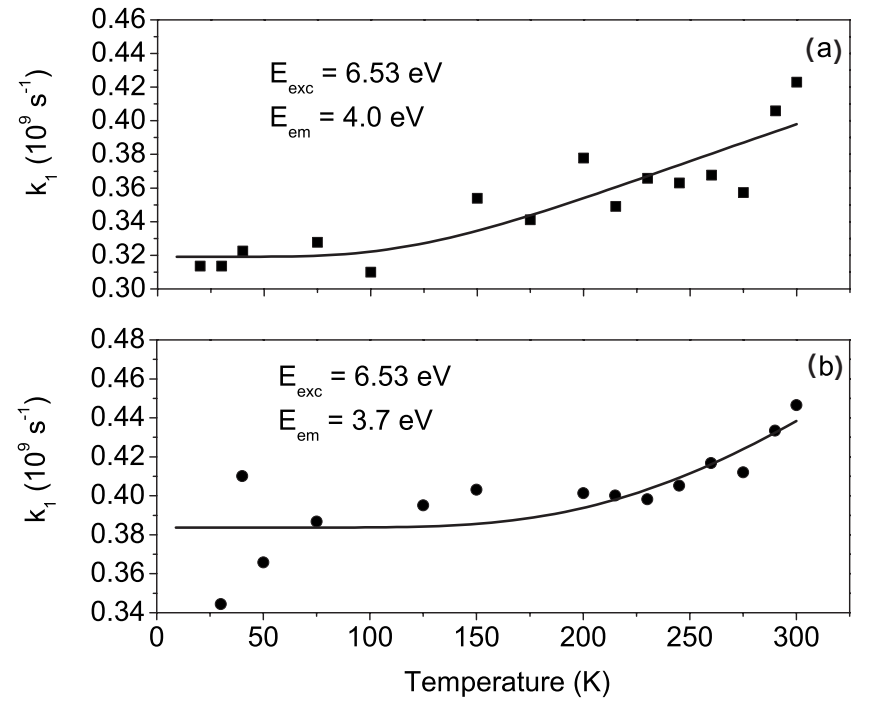

FIG. 9. Temperature dependence of the fast component decay rate as deduced by the biexponential model monitoring the emission at $3.7 \mathrm{eV}$ (a) and $4.0 \mathrm{eV}$ (b) excited at $6.53 \mathrm{eV}$ in samples A. Solid lines are fitting results according to Eqs. (4) and (5) (see text for details).

properties were investigated in samples A by collecting the PL amplitude at the emission peaks of the two centers. The data can be fitted by assuming an one-process quenching model according to the following expression: ${ }^{29,30}$

$$
I(T)=\frac{I_{0}}{1+U \exp \left(-E_{a} / k_{B} T\right)},
$$

where $I(T)$ is the PL amplitude, $E_{a}$ is the activation energy, and $U$ is the preexponential factor. The activation energy estimated by fitting the PL amplitude at $3.7 \mathrm{eV}$ excited at $5.39 \mathrm{eV}$ is $(29 \pm 1) \mathrm{meV}$ (square correlation factor $R^{2} \geqslant 0.99$ ). In the case of the PL amplitude at $4.0 \mathrm{eV}$ under $6.53 \mathrm{eV}$ excitation the estimated activation energies is $(37 \pm 3) \mathrm{meV}$ (square correlation factor $\left.R^{2} \geqslant 0.99\right)$. We also calculated the activation energy at different emissions of the UV PL band in the range $3.2-4.2 \mathrm{eV}$, and we did not observe any distribution of the reported value. On the contrary, the activation energy in the case of samples B shows a distribution of the calculated values as a function of the selected emission energy of the UV PL band (see inset of Fig. 6). The calculated values display a maximum of about $150 \mathrm{meV}$ around the emission at $3.5 \mathrm{eV}$ (excitation energy $5.64 \mathrm{eV}$ ), the activation energy at $3.7 \mathrm{eV}$ being $(120 \pm 20) \mathrm{meV}$ (square correlation factor $R^{2} \geqslant 0.97$ ).

In order to discuss and compare the different values of activation energies calculated with the two methods, we follow a different approach. Since the two UV emission bands largely overlap, we gathered and analyzed the PL amplitude integrated over a limited bandwidth $(5 \mathrm{~nm})$. This approach allowed us to experimentally separate the contribution of the two bands and to investigate the distribution of the activation energy all over the emission band. A different approach consists in recording the whole UV emission at different temperatures and excitation energies and deconvolving the spec- tra with Gaussian bands. The evaluation of the activation energy is then carried out on the temperature dependence of the integrated PL amplitude of each band. The deconvolution of the spectra is performed with three Gaussian bands, two bands for the two UV emitting centers and the third one to heuristically account for the emission around $2.7 \mathrm{eV}$, as previously reported. ${ }^{23}$ This approach confirmed the activation energies previously calculated. In particular, in the case of samples A the evaluated activation energy of the PL band at $3.7 \mathrm{eV}$ is $(38 \pm 9) \mathrm{meV}$ (excitation energy $6.53 \mathrm{eV}, R^{2}$ $\geqslant 0.89)$ and $(90 \pm 10) \mathrm{meV}$ in the case of samples B (excitation energy $\left.5.64 \mathrm{eV}, R^{2} \geqslant 0.96\right)$. The procedure described allowed to evaluate also the activation energy of the emission bands at $4.0 \mathrm{eV}$ and at about $2.7 \mathrm{eV}$. However, the value of $E_{a}$ of the $4.0 \mathrm{eV}$ band in samples $\mathrm{A}$ is underestimated $\left(E_{a}\right.$ $\left.=14 \pm 4 \mathrm{meV}, R^{2} \geqslant 0.83\right)$ and in samples $\mathrm{B}$ is affected by a large uncertainty $\left(E_{a}=30 \pm 20 \mathrm{meV}, R^{2} \geqslant 0.68\right)$ because of the small relative contribution of this band to the overall emission, a contribution which decreases as the temperature decreases. ${ }^{23}$ To complete the analysis we evaluate the activation energy of the band around $2.7 \mathrm{eV}: 60 \pm 20 \mathrm{meV}\left(R^{2}\right.$ $\geqslant 0.87)$ and $43 \pm 3 \mathrm{meV}\left(R^{2} \geqslant 0.98\right)$ in samples $\mathrm{A}$ and $\mathrm{B}$, respectively.

To summarize the results here reported we conclude that the two proposed analysis of the temperature dependence of the PL amplitude confirmed the activation energy of the $4.0 \mathrm{eV}$ band of about 30-40 meV for both samples A and B, in good agreement with the value calculated by the temperature dependence of the decay rate of the fast component. The estimated value of the activation energy of the 3.7-eV band is different in the two samples, being $20-40 \mathrm{meV}$ in samples A and 90-120 meV in samples B. In addition a distribution of the reported value is observed in samples B as a function of the monitored emission energy within the overall band, while this effect is not observed in samples A. The different value of the activation energy of the 3.7-eV band in samples with different porosity explains the different relative weight of the two UV-emitting bands as a function of the temperature, previously reported. ${ }^{23}$ Indeed, not only the amount of different centers is different in samples with small and large pore diameters, but also their quenching properties are different, causing the observed variation in the relative contributions of the centers. In small-pore-diameter samples the large activation energy of the $3.7-\mathrm{eV}$ band favors the latter with respect to the $4.0-\mathrm{eV}$ band as the temperature decreases. On the contrary, in samples with large pore diameter the two bands show comparable activation energies and the increase of the $4.0-\mathrm{eV}$ band with respect to the $3.7-\mathrm{eV}$ one at low temperatures can be interpreted in terms of an energy transfer mechanism. The different activation energy of the same center in samples with different pore diameters can be ascribed to the thermal history of the samples. ${ }^{32,33}$ Indeed, according to the synthesis procedure, ${ }^{26}$ different thermal treatments were carried out to achieve samples with different and controlled porosity.

A final comment pertains the activation energy of the $4.0-\mathrm{eV}$ band evaluated by means of the analysis of the time decay by monitoring the emission at $3.7 \mathrm{eV}$. Indeed, the reported value is larger than the previously reported ones. A possible explanation is related to the presence of the band at 
$2.7 \mathrm{eV}$. This band shows a composite time decay with a fast component comparable to that of the $4.0-\mathrm{eV}$ band. ${ }^{31}$ The blue band largely overlaps the $3.7-\mathrm{eV}$ band, especially in the case of large-pore-diameter samples where its activation energy is of about $60 \mathrm{meV}$. When monitoring the emission at $3.7 \mathrm{eV}$ the contribution of the blue band and that of the 4.0-eV band are comparable and the former increases as the temperature decreases. We can speculate that the larger values of calculated activation energy can be due to the effect of the presence of the $2.7-\mathrm{eV}$ band.

\section{CONCLUSIONS}

The thermal quenching properties of mesoporous silica were discussed by analyzing the temperature dependence of the lifetimes of the UV-emitting centers and of the PL amplitudes. The reported analysis allows us to estimate the activation energies of the 3.7- and 4.0-eV bands in samples with different pore diameters. We found that the activation energy of the $3.7-\mathrm{eV}$ band is $30-40 \mathrm{meV}$ in samples with large pore diameter and $90-120 \mathrm{meV}$ in samples with small pore diameter. The activation energy of the $4.0-\mathrm{eV}$ band is $30-40 \mathrm{meV}$ for both samples. Reported data allowed us to clarify the different distribution of the centers as the temperature decreases observed in samples with different pore diameters.

\section{ACKNOWLEDGMENTS}

We thank M. Kirm of the G. Zimmerer group and A. Paleari for the SR experimental time at DESY (Hamburg). This work was supported by the European CommunityResearch Infrastructure Action under the FP6 "Structuring the European Research Area" Program through the Integrated Infrastructure Initiative "Integrating Activity on Synchrotron and Free Electron Laser Science." This study was also supported by a national research project (PRIN2002) of MIUR (Ministero dell'Università e della Ricerca).
*Corresponding author. FAX +39070510171 cm.carbonaro@dsf.unica.it

${ }^{1}$ A. G. Cullis, L. T. Canham, and P. D. Calcot, J. Appl. Phys. 82, 909 (1997).

${ }^{2}$ A. G. Cullis and L. T. Canham, Nature (London) 353, 335 (1991).

${ }^{3}$ H. Koyama and N. Koshida, Solid State Commun. 103, 37 (1997)

${ }^{4}$ A. J. Kontkiewicz, A. M. Kontkiewicz, J. Siejka, S. Sen, G. Novak, A. M. Hoff, P. Sakthivel, K. Ahmed, P. Mukherjee, S. Witanachchi, and J. Lagowski, Appl. Phys. Lett. 65, 1346 (1994).

${ }^{5}$ N. Sagawa and T. Uchino, Appl. Phys. Lett. 87, 251923 (2005).

${ }^{6}$ T. Uchino, N. Kurumoto, and N. Sagawa, Phys. Rev. B 73, 233203 (2006).

${ }^{7}$ Y. D. Glinka, S. H. Lin, L. P. Hwang, and Y. T. Chen, Appl. Phys. Lett. 77, 3968 (2000).

${ }^{8}$ Y. D. Glinka, A. S. Zyubin, A. M. Mebel, S. H. Lin, L. P. Hwang, and Y. T. Chen, Eur. Phys. J. D 16, 279 (2001).

${ }^{9}$ H. J. Chang, Y. F. Chen, H. P. Lin, and C. Y. Mou, Appl. Phys. Lett. 78, 3791 (2001).

${ }^{10}$ M. Zhang, E. Ciocan, Y. Bando, K. Wada, L. L. Cheng, and P. Pirouz, Appl. Phys. Lett. 80, 491 (2002).

${ }^{11}$ A. Monnier, F. Sshuth, Q. Huo, D. Kumar, D. Margolese, R. S. Maxwell, G. D. Stucky, M. Krishanamutry, P. Petroff, A. Firouzi, M. Janicke, and B. F. Chmelka, Science 261, 1299 (1993).

${ }^{12}$ Y. D. Glinka, S. H. Lin, and Y. T. Chen, Phys. Rev. B 62, 4733 (2000).

${ }^{13}$ Y. D. Glinka, S. H. Lin, L. P. Hwang, Y. T. Chen, and N. H. Tolk, Phys. Rev. B 64, 085421 (2001).

${ }^{14}$ Y. D. Glinka, S. H. Lin, and Y. T. Chen, Phys. Rev. B 66, 035404 (2002).

${ }^{15}$ Y. D. Glinka, S. N. Naumenko, V. M. Ogenko, and A. A. Chuiko, Opt. Spectrosc. 71, 250 (1991).

${ }^{16}$ H. Koyama, Y. Matsushita, and N. Koshida, J. Appl. Phys. 83, 1776 (1998).
${ }^{17}$ Y. D. Glinka, S. H. Lin, and Y. T. Chen, Appl. Phys. Lett. 75, 778 (1999).

${ }^{18}$ G. G. Qin, J. Lin, J. Q. Duan, and G. Q. Yao, Appl. Phys. Lett. 69, 1689 (1996).

${ }^{19}$ H. Z. Song, X. M. Bao, N. S. Li, and X. L. Wu, Appl. Phys. Lett. 72, 356 (1998).

${ }^{20}$ N. Chiodini, F. Meinardi, F. Morazzoni, A. Paleari, R. Scotti, and D. Di Martino, Appl. Phys. Lett. 76, 3209 (2000).

${ }^{21}$ B. Yao, H. Shi, X. Zhang, and L. Shang, Appl. Phys. Lett. 78, 174 (2001).

${ }^{22}$ A. Anedda, C. M. Carbonaro, F. Clemente, R. Corpino, and P. C. Ricci, J. Phys. Chem. B 109, 1239 (2005).

${ }^{23}$ C. M. Carbonaro, F. Clemente, R. Corpino, P. C. Ricci, and A. Anedda, J. Phys. Chem. B 109, 14441 (2005).

${ }^{24}$ A. Anedda, C. M. Carbonaro, F. Clemente, R. Corpino, S. Grandi, P. Mustarelli, and A. Magistris, J. Non-Cryst. Solids 322, 68 (2003).

${ }^{25}$ A. Anedda, C. M. Carbonaro, F. Clemente, R. Corpino, and P. C. Ricci, J. Phys. Chem. B 107, 13661 (2003).

${ }^{26}$ J.-L. Nogues, C. Balaban, and W. V. Moreshead, U.S. Patent No. 50,76,980 (31 March 1991).

${ }^{27}$ G. Zimmerer, Nucl. Instrum. Methods Phys. Res. A 308, 178 (1991).

${ }^{28}$ J. R. Lakowicz, Principles of Fluorescence Spectroscopy, 2nd ed. (Kluver Academic, New York, 1999).

${ }^{29}$ D. Bimberg, M. Sondergeld, and E. Grobe, Phys. Rev. B 4, 3451 (1971).

${ }^{30}$ T. D. Culp, U. Hömmerich, J. M. Redwing, T. F. Kuench, and K. L. Bray, J. Appl. Phys. 82, 368 (1997).

${ }^{31}$ A. Anedda, C. M. Carbonaro, F. Clemente, R. Corpino, and P. C. Ricci, J. Non-Cryst. Solids 351, 1924 (2005).

${ }^{32}$ J. M. Garcia, M. A. Mondragon, C. S. Tellez, A. Campero, and V. M. Castaño, Mater. Chem. Phys. 41, 15 (1995).

${ }^{33}$ J. Zhang and J. Lin, Microporous Mater. 75, 115 (2004). 